\title{
Dynamic criticality in glass-forming liquids
}

\author{
Stephen Whitelam, ${ }^{1}$ Ludovic Berthier, ${ }^{1,2}$ and Juan P. Garrahan ${ }^{1,3}$ \\ ${ }^{1}$ Theoretical Physics, University of Oxford, 1 Keble Road, Oxford, OX1 3NP, UK \\ ${ }^{2}$ Laboratoire des Verres, Université Montpellier II, 34095 Montpellier, France \\ ${ }^{3}$ School of Physics and Astronomy, University of Nottingham, Nottingham, NG7 2RD, UK
}

(Dated: October 29, 2018)

\begin{abstract}
We propose that the dynamics of supercooled liquids and the formation of glasses can be understood from the existence of a zero temperature dynamical critical point. To support our proposal, we derive a dynamic field theory for a generic kinetically constrained model, which we expect to describe the dynamics of a supercooled liquid. We study this field theory using the renormalization group (RG). Its long time behaviour is dominated by a zero temperature critical point, which for $d>2$ belongs to the directed percolation universality class. Molecular dynamics simulations seem to confirm the existence of dynamic scaling behaviour consistent with the RG predictions.
\end{abstract}

PACS numbers: 64.60.Cn, 47.20.Bp, 47.54.+r, 05.45.-a

The recipe for making a glass is simple [1]: rapidly cool a liquid through its melting point to avoid crystallization. Cool it further, and the liquid eventually becomes so viscous that it forms a non-crystalline solid, or glass. Glasses are common in nature, but our theoretical understanding of their formation is poor [2]. Here we offer analytical and numerical results to support the proposition that the dynamics of glass-forming supercooled liquids is controlled by a zero temperature dynamic critical point.

Our starting point is the real-space description of supercooled liquids studied in 3, 4 and based on ideas and models originally proposed in 5, , 6, 7]. In this approach the phenomenon of dynamic heterogeneity [8, 9, 10] plays a central role. The link between dynamic heterogeneity and glass formation is the subject of much current research. If this link is verified it will be an indication that the slow dynamics of glass formers is governed by dynamic spatial fluctuations (see [1, 12] for alternatives based on thermodynamics), in contrast with the assumption of homogeneity of mode coupling theories [13, 14].

The microscopic coarse-grained approach of $[3$, 4] relies on two observations: (i) at low temperature very few particles are mobile, and these mobility excitations are localized in space; (ii) mobile regions are needed to allow neighbouring regions to themselves become mobile. This is the concept of dynamic facilitation [5, 7]. We show that this picture can be cast as a dynamical field theory and its scaling behaviour derived from a dynamic renormalization group (RG) analysis. We find that scaling properties are determined by a zero temperature critical point. For the simple case we consider, and for $d>2$, this critical point is that of directed percolation (DP) [15]. We also show, by performing extensive molecular dynamics simulations, that supercooled Lennard--Jones binary mixtures display scaling behaviour consistent with that predicted by RG.

We build an effective microscopic model as follows. A supercooled fluid in $d$ spatial dimensions is coarse-grained into cells of linear size of the order of the static correlation length as given by the pair correlation function. Cells are labeled by a scalar mobility field, $n_{i}$, identified by coarsegraining the system on a microscopic time scale. Mobile regions carry a free energy cost, and when mobility is low interactions between cells are not important. Adopting a thermal language, we expect static equilibrium to be determined by the non-interacting Hamiltonian [7],

$$
H=\sum_{i=1}^{N} n_{i} .
$$

At low mobility, the distinction between single and multiple occupancy is probably irrelevant. We assume the latter case for technical simplicity. The dynamics of the mobility field is given by a master equation,

$$
\partial_{t} P(\{n\}, t)=\sum_{i} \mathcal{C}_{i}(\{n\}) \hat{\mathcal{L}}_{i} P(\{n\}, t),
$$

where $P(\{n\}, t)$ is the probability that the system has configuration $\{n\}$ at time $t$. The local operators $\hat{\mathcal{L}}_{i}$ encode the existence of local quanta of mobility. For nonconserved dynamics they describe the creation and destruction of mobility at site $i$,

$$
\begin{aligned}
\hat{\mathcal{L}}_{i} P\left(n_{i}, t\right)= & \gamma\left(n_{i}+1\right) P\left(n_{i}+1, t\right)+\rho P\left(n_{i}-1, t\right) \\
& -\left(\gamma n_{i}+\rho\right) P\left(n_{i}, t\right),
\end{aligned}
$$

where the dependence of $P$ on cells other than $i$ has been suppressed. The rates for mobility destruction, $\gamma$, and creation, $\rho$, are chosen so that (2) obeys detailed balance with respect to (1) at low temperature, $\rho / \gamma=e^{-1 / T} \approx c$, with $c \equiv\left\langle n_{i}\right\rangle$; the brackets indicate a thermal average. The average concentration of excitations $c$ is the control parameter of the problem. $\mathcal{C}_{i}(\{n\})$ is the kinetic constraint designed to suppress the dynamics of cell $i$ if surrounded by immobile regions. It cannot depend on $n_{i}$ itself if (2) is to satisfy detailed balance. To reflect the local nature of dynamic facilitation we allow $\mathcal{C}_{i}$ to depend only on the nearest neighbours of $i[7]$ and require that $\mathcal{C}_{i}$ is small when local mobility is scarce. 
The large time and length scale behaviour of the model defined by Eqs. (11)-(3) is derived from the analysis of the corresponding field theory. The technique to recast the master equation (3) as a field theory is standard [16, 17], so we only outline the procedure. One introduces a set of bosonic creation and annihilation operators for each site $i, a_{i}^{\dagger}$ and $a_{i}$, satisfying $\left[a_{i}^{\dagger}, a_{j}\right]=\delta_{i j}$, and passes to a Fock space defined by a state vector $|\Psi(t)\rangle \equiv \sum_{\left\{n_{i}\right\}} p\left(n_{1}, n_{2}, \ldots, t\right) a_{1}^{\dagger n_{1}} a_{2}^{\dagger n_{2}} \ldots|0\rangle$. The master equation (3) then assumes the form of a Euclidean Schrödinger equation, $d|\Psi(t)\rangle / d t=-\hat{H}|\Psi(t)\rangle$, with $\hat{H}=\sum_{i} \hat{\mathcal{C}}_{i}\left(\left\{a_{j}^{\dagger} a_{j}\right\}\right) \hat{H}_{i}^{(0)}$. The unconstrained piece $\hat{H}_{i}^{(0)}$ reads

$$
\hat{H}_{i}^{(0)}=-\gamma\left(a_{i}-a_{i}^{\dagger} a_{i}\right)-\rho\left(a_{i}^{\dagger}-1\right) .
$$

The Hamiltonian (4) describes the creation and destruction of bosonic excitations with rates $\rho$ and $\gamma$. The evolution operator $e^{-\hat{H} t}$ can then be represented as a coherent state path integral 17] weighted by the dynamical action

$$
\mathcal{S}\left[\phi_{i}^{\star}, \phi_{i}, t_{0}\right]=\sum_{i} \int_{0}^{t_{0}} d t\left(\phi_{i}^{\star} \partial_{t} \phi_{i}+H_{i}\left(\phi^{\star}, \phi\right)\right),
$$

where we have suppressed boundary terms coming from the system's initial state vector. The fields $\phi_{i}^{\star}(t)$ and $\phi_{i}(t)$ are the complex surrogates of $a_{i}^{\dagger}$ and $a_{i}$, respectively, while $H_{i}$ has the same functional form as (4) with the bosonic operators replaced by the complex fields. At the level of the first moment we have $\left\langle n_{i}\right\rangle=\left\langle\phi_{i}\right\rangle$, so we may regard $\phi_{i}$ as the complex mobility field. The last step in the passage to a field theory is to take the continuum limit, $\sum_{i} \rightarrow a^{-d} \int d^{d} x, \phi_{i}(t) \rightarrow a^{d} \phi(\mathbf{x}, t)$, and $\phi_{i}^{\star}(t) \rightarrow \phi^{\star}(\mathbf{x}, t)$, where $a$ is the lattice parameter.

The definition of the model is completed by specifying the functional form of the kinetic constraint. The simplest non-trivial form is the isotropic facilitation function, $\mathcal{C}_{i}=\sum n_{j}$, where the sum is over nearest neighbours of site $i$. With this choice we expect the one-spin facilitated Fredrickson-Andersen (FA) model in $d$ dimensions [5, 7] to be in the same universality class as our model. Different choices for the operators $\hat{H}^{(0)}$ and $\mathcal{C}$ lead to field theoretical versions of more complicated facilitated models. A diffusive $\hat{H}^{(0)}$, for example, would correspond to a constrained lattice gas like that of Kob and Andersen [7, 18]; an asymmetric $\mathcal{C}$, to the East model [6, 7] and its generalizations. The dynamic action finally reads

$$
\begin{aligned}
& \mathcal{S}\left[\bar{\phi}, \phi, t_{0}\right]=\int d^{d} x \int_{0}^{t_{0}} d t\left(\bar{\phi}\left(\partial_{t}-D_{0} \nabla^{2}-\kappa_{0}^{(m)}\right) \phi\right. \\
& \quad+\bar{\phi} \phi\left(\lambda_{0}^{(1)}+\nu_{0}^{(1)} \nabla^{2}\right) \phi+\bar{\phi} \phi\left(\lambda_{0}^{(2)}+\nu_{0}^{(2)} \nabla^{2}\right) \bar{\phi} \phi \\
& \left.\quad-\bar{\phi} \phi\left(\kappa_{0}^{(v)}+\sigma_{0} \nabla^{2}\right) \bar{\phi}\right)
\end{aligned}
$$

where we have omitted higher-order gradient terms, and suppressed boundary contributions coming from initial and projection states. We also made the shift $\phi^{\star}=1+$ $\bar{\phi}$ [17], and defined $\lambda_{0}^{(1,2)} \equiv 2 d a^{d} \gamma, \kappa_{0}^{(m, v)} \equiv 2 d \rho, \nu_{0}^{(1,2)} \equiv$ $\gamma a^{d+2}, \sigma_{0} \equiv a^{2} \rho$. We defined $D_{0} \equiv \sigma_{0}$ to emphasise the emergence of a diffusive term, although in the unshifted model there is no purely diffusive process. The action (6) is the starting point for our RG analysis. We will leave the technical details to a later paper [19] and here state only the most important conclusions.

The action (6) has the form of a single species branching and coalescing diffusion-limited reaction with additional momentum dependent terms [15]. By integrating out the response field, we obtain a Langevin equation for the evolution of $\phi$. This equation has a critical point at $c=0$, i.e. $T=0$, describing the crossover from an exponential decay of mobility at finite $c$, i.e. $T>0$, to an algebraic decay at $c=0$. In the absence of noise (corresponding to neglecting terms quadratic in $\bar{\phi}$ ) we find that (6) admits the Gaussian exponents $\left(\nu_{\perp}^{G}, \nu_{\|}^{G}, \beta^{G}\right)=\left(\frac{1}{2}, 1,1\right)$. Here $\nu_{\perp}$ and $\nu_{\|}$control the growth of spatial $\left(\xi_{\perp}\right)$ and temporal $\left(\xi_{\|}\right)$length scales near criticality, $\xi_{\perp} \sim c^{-\nu_{\perp}}$ and $\xi_{\|} \sim c^{-\nu_{\|}}$, while $\beta$ governs the long-time scaling of the density, $n \sim c^{\beta}$.

These Gaussian power laws are modified by fluctuations which we treat using the RG. Identifying the unphysical microscopic singularities present in (6) as a consequence of taking the continuum limit, one invokes scale invariance and dimensional analysis to extract the macroscopic scaling; see Fig. 10 We find the following.

(i) The critical point remains at $c=0$. There is thus no finite temperature phase transition. For finite $T$, and at asymptotically long length and time scales, the system will therefore exhibit Gaussian power laws.

(ii) Dimensional analysis shows that the upper critical dimension of the model is $d_{c}=4$. For $d \leq 4$ we account for fluctuations by studying the behaviour of the effective couplings. These couplings come from diagrams like in Fig. 1b. For $2<d \leq 4$ we find that only the dimensionless coupling $x \equiv A_{d} \kappa^{(v)} \lambda^{(1)} \mu^{-\epsilon} / D^{2}$ matters, where $A_{d} \equiv 2^{2-d} \pi^{-d / 2} \Gamma(3-d / 2), \epsilon \equiv 4-d$, and $\mu$ is an external momentum scale. The system exhibits scale invariance at the fixed point $x^{*}=2 \epsilon / 3$, and all other effective couplings are irrelevant, both at this and at the Gaussian fixed point, so that all interaction terms in (6) except for $\lambda^{(1)}$ and $\kappa^{v}$ are irrelevant. It follows that our system is described for intermediate length and time scales by the directed percolation (DP) critical point and its associated power laws 15. To order $O(\epsilon)$ they are $\left(\nu_{\perp}, \nu_{\|}, \beta\right)=\left(\frac{1}{2}+\frac{\epsilon}{16}, 1+\frac{\epsilon}{12}, 1-\frac{\epsilon}{6}\right)$.

(iii) For $d \leq 2$ one cannot access the system's behaviour by way of a perturbation expansion around $d=4$ because newly relevant terms in the action introduce divergences to all orders in perturbation theory. We do not expect DP behaviour, therefore.

Physically, RG identifies the relevant microscopic interactions in $d=3$ and shows that the action (6) re- 
(a)
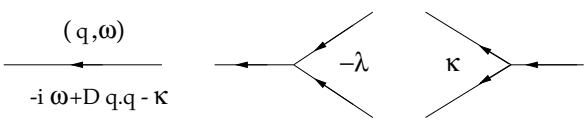

(c)

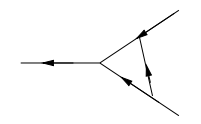

(b)

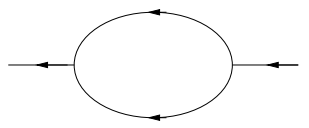

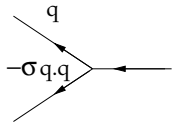

FIG. 1: Elements of the RG calculation. (a) Gaussian prop-
agator and vertices in the frequency-wavevector domain corresponding to the terms in the dynamic action (6); time runs from right to left. (b) Structure of diagrams dictating the effective couplings. (c) A diagram contributing to the renormalization of the effective coupling $x$.

sembles that of DP in the case where the coupling constant for particle self-destruction vanishes. This is why the critical point is at $T=0$, so that finite $T$ dynamics takes place in the active phase of DP [15]. Moreover, simulations of the $3 d$ FA model also confirm the DP picture 19. For example, for the correlation timescale we expect $(D \tau) \sim(c \tau) \sim c^{-\nu_{\|}}$, so that $t \sim c^{-\delta}$ with $\delta=1+\nu_{\|}=2+\epsilon / 12$. The numerics indeed shows that $\delta \approx 2.10$ [19], rather than the naive estimate $\delta=$ $1+2 / d \approx 1.66[7]$.

Our analysis implies that the slowdown is a dynamical critical slowing down as the critical point is approached from above. Correlation time and length scales grow as inverse powers of $c$. Thermal activation results from $c \sim e^{-1 / T}$. Dynamical scaling is predicted to occur when the dynamic correlation length becomes appreciably larger than the lattice spacing. This happens therefore for temperatures lower than $T_{o}$, the onset temperature for dynamic heterogeneity [4, 21].

Our analytical results apply to systems with isotropic facilitation. We therefore expect them to apply to strong liquids, and to those which exhibit a crossover from fragile to strong behaviour [3]. While we do not expect DP behaviour for the case of anisotropic constraints or conserved order parameters [19], we do expect a zerotemperature critical point to be the generic feature of both strong and fragile glass formers [19, 20]. We expect that the scaling properties of liquids in their fragile regime can be described by the field theory for a facilitated model with directional persistence, such as the East model [19].

The field theory suggests the following physical picture. The viscosity of a supercooled liquid increases rapidly as $T$ is lowered, because the dynamics becomes increasingly spatially correlated. A glass is obtained when the liquid's relaxation time exceeds the experimental time scale. The scaling properties of time and length scales and therefore the physical properties of supercooled liquids are governed by a zero temperature dynamic critical point.

As shown in [3, 4], this picture accounts quantitatively for several observations concerning thermodynamic, dynamic, topographic and spatial properties of supercooled liquids. However, the existence of critical fluctuations and dynamic scaling remains to be proven 22, 23. We now present numerical evidence to this end.

We have performed molecular dynamics simulations of a well-characterized model for supercooled liquids, the canonical 80:20 binary Lennard-Jones mixture defined in Ref. 24]; further numerical details are found in 23]. According to the above analysis, power law spatial correlations develop in the dynamics of a supercooled liquid when $T$ is lowered. With this in mind, we have measured $S_{\mathbf{k}}(\mathbf{q}, T)$, defined as the spatial Fourier transform of the following two-point, two-time correlation function,

$$
C_{\mathbf{k}}(\mathbf{r})=\frac{\left\langle F_{\mathbf{k}}(\mathbf{0}, \tau) F_{\mathbf{k}}(\mathbf{r}, \tau)\right\rangle-\left\langle F_{\mathbf{k}}(\mathbf{r}, \tau)\right\rangle^{2}}{\left\langle F_{\mathbf{k}}(\mathbf{r}, \tau)^{2}\right\rangle-\left\langle F_{\mathbf{k}}(\mathbf{r}, \tau)\right\rangle^{2}},
$$

where $F_{\mathbf{k}}(\mathbf{r}, t)=\sum_{j} \delta\left(\mathbf{r}_{j}(0)-\mathbf{r}\right) \cos \left(\mathbf{k} \cdot\left[\mathbf{r}_{j}(t)-\mathbf{r}_{j}(0)\right]\right)$ is a natural local indicator of the dynamics of the liquid. In these expressions $\mathbf{r}_{j}(t)$ is the position of particle $j$ at time $t$, and $\tau=\tau(\mathbf{k}, T)$ is defined in a standard way from the time decay of $\left\langle F_{\mathbf{k}}(\mathbf{r}, t)\right\rangle$. Similar functions replacing density correlations by particle overlap or velocity correlations have been discussed [9, 10, 22]. As predicted theoretically, we find that the $\mathbf{q}$ dependence of $S_{\mathbf{k}}$ is well described by the following scaling form,

$$
S_{\mathbf{k}}(\mathbf{q}, T) \simeq \ell_{\mathbf{k}}^{2-\eta} \mathcal{S}\left(q \ell_{\mathbf{k}}\right),
$$

for all $\mathbf{k}$, although a precise determination of $\eta$ has proven impossible because it is small, as is found in the RG approach. We are aware of no alternative theoretical prediction for the correlator (7). The scaling function $\mathcal{S}(x)$ behaves as $\mathcal{S}(x \rightarrow 0)=$ const, $\mathcal{S}(x \rightarrow \infty) \sim x^{\eta-2}$. Moreover, it is universal in the sense that it appears to be independent of $\mathbf{k}$ for a range of inverse wavevectors from the interparticle distance up to the size of the simulation box. From (8), we conclude that universal power law correlations develop in supercooled liquids. Imposing $\eta=0$, we can use Eq. (8) to numerically extract the length scale $\ell_{\mathbf{k}}(T)$, which grows when $T$ decreases, as in 22].

The above RG analysis predicts scaling laws for length and time scales expressed as a function of $c$, the concentration of mobile regions. This quantity is difficult to measure in simulations. However, one can eliminate $c$ in favour of $\ell \sim \tau^{1 / z}$, which defines a dynamic exponent $z=\delta / \nu_{\perp}$. Similarly, it is convenient to measure $\chi_{\mathbf{k}}(T) \equiv S_{\mathbf{k}}(\mathbf{q}=\mathbf{0}, T)$, which is predicted to scale as $\chi \sim \tau^{1 / \Delta}, \Delta^{-1}=(2-\eta) \nu_{\perp} / \delta$. These two scaling predictions are tested in Fig. 2] We find that the power law scalings anticipated theoretically are well satisfied. We find also that the exponents $z$ and $\Delta$ that we measure from Fig. 22 do not depend on the chosen physical observable. We note that the numerical values of the exponents are in reasonable agreement with the RG predictions, despite the fact that the simulated liquid is thought to be 


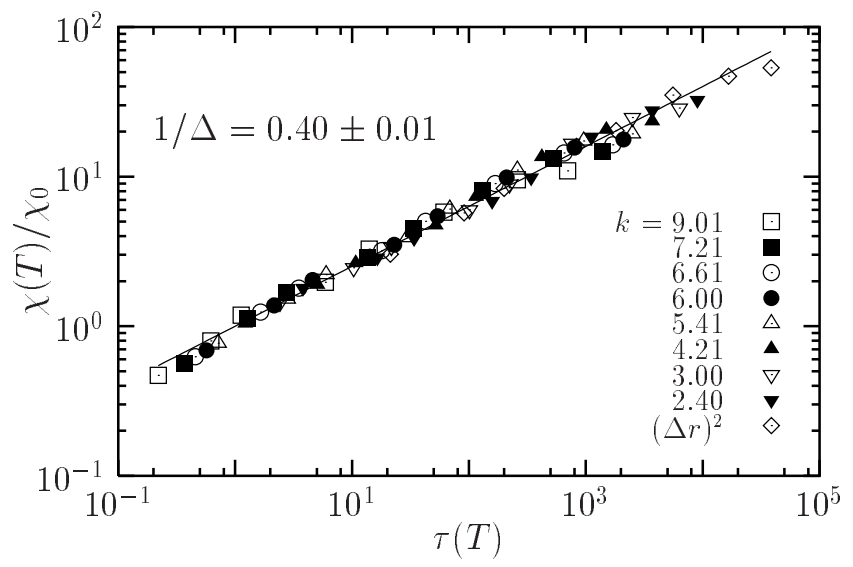

2002-01927 (EU), the Glasstone Fund, CNRS France, Linacre and Worcester Colleges Oxford, and numerical support from the Oxford Supercomputing Centre.

[1] C.A. Angell, Science 267, 1924 (1995).

[2] M.D. Ediger, C.A. Angell and S.R. Nagel, J. Phys. Chem. 100, 13200 (1996); P.G. Debenedetti and F.H. Stillinger, Nature 410, 259 (2001).

[3] J.P. Garrahan and D. Chandler, Phys. Rev. Lett. 89, 035704 (2002); Proc. Natl. Acad. Sci. USA 100, 9710 (2003).

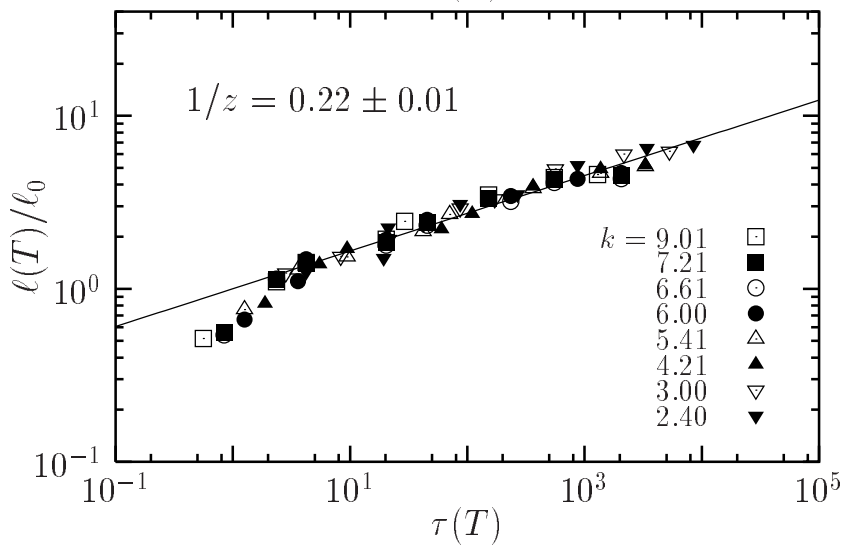

[4] L. Berthier and J.P. Garrahan, J. Chem. Phys. 119, 4367 (2003); Phys. Rev. E 68, 041201 (2003).

[5] S.H. Glarum, J. Chem. Phys. 33, 639 (1960); G.H. Fredrickson and H.C. Andersen, Phys. Rev. Lett. 53, 1244 (1984); R.G. Palmer et al., ibid. 958 (1984).

[6] J. Jäckle and S. Eisinger, Z. Phys. B 84, 115 (1991).

[7] F. Ritort and P. Sollich, Adv. in Phys. 52, 219 (2003).

[8] See e.g., K. Schmidt-Rohr and H. Speiss, Phys. Rev. Lett. 66, 3020 (1991); M.T. Cicerone and M.D. Ediger, J. Chem. Phys. 103, 5684 (1995); E.V. Russell et al., Phys. Rev. Lett. 81, 1461 (1998); L.A. Deschenes and D.A. Vanden Bout, Science 292, 255 (2001); E. Weeks et al., Science 287, 627 (2000).

[9] See e.g., T. Muranaka and Y. Hitawari, Phys. Rev. E 51, R2735 (1995); D. Perera and P. Harrowell, Phys. Rev. E 51, 314 (1995); B. Doliwa and A. Heuer, Phys. Rev. Lett. 80, 4915 (1998); C. Donati et al., Phys. Rev. E 60, 3107 (1999).

FIG. 2: Dynamic scaling of the susceptibility (top) and the correlation length (bottom) measured for various wavevectors in the binary Lennard-Jones mixture; $(\Delta r)^{2}$ indicates the $k \rightarrow 0$ limit. For each $\mathbf{k}$, we independently measure $\chi_{\mathbf{k}}$, $\ell_{\mathbf{k}}$ and $\tau_{\mathbf{k}}$ for $T \in[0.45 ; 2.0]$. The parameters $\chi_{0}$ and $\ell_{0}$ are determined as the numerical prefactors in power law fits, so that the universal dynamic scaling observed in these plots is obtained without free parameters. Errorbars reflect observable to observable fluctuations of the exponents, and do not take into account systematic errors. The few points outside the power law in the bottom figure are all for $T=2.0$, much above the onset of slow dynamics, $T_{o} \simeq 1.0$.

fragile. A more precise comparison between theory and simulation would require a better finite size scaling analysis [25]. Note finally that [14] indirectly predicts a similar scaling between $\chi$ and $\tau$ although neither the value of the exponent nor its relation to spatial structures were derived.

In conclusion, this paper presents further [3, 4] theoretical evidence that the slow dynamics in supercooled liquids is governed by a zero temperature dynamic critical point at which time and length scales diverge. We propose that this critical point is responsible for the existence of the glass state.

We thank J.L. Cardy, D. Chandler, M. Kardar, and O. Zaboronski for useful discussions. We acknowledge financial support from EPSRC Grants No. GR/R83712/01 and GR/S54074/01, Marie Curie Grant No. HPMF-CT-

[10] H. Sillescu, J. Non-Cryst. Solids 243, 81 (1999); M.D. Ediger, Annu. Rev. Phys. Chem. 51, 99 2000; S.C. Glotzer, J. Non-Cryst. Solids, 274, 342 (2000).

[11] Kivelson D. et al., Physica A 219, 27 (1995).

[12] X. Xia and P.G. Wolynes, Proc. Natl. Acad. Sci. USA 97, 2990 (2000).

[13] W. Götze and L. Sjögren, Rep. Prog. Phys. 55, 55 (1992).

[14] S. Franz and G. Parisi, J. Phys. C 12, 6335 (2000).

[15] H. Hinrichsen, Adv. Phys. 49, 815 (2000).

[16] M. Doi, J. Phys. A 9, 1465 (1976); L. Peliti, J. Physique 46, 1469, (1985).

[17] B.P. Lee and J.L. Cardy, J. Stat. Phys. 80, 971, (1995); J.L. Cardy and U.C. Täuber, J. Stat. Phys. 90, 1, (1998).

[18] W. Kob and H.C. Andersen, Phys. Rev. E 48, 4364 (1993).

[19] S. Whitelam, L. Berthier and J.P. Garrahan, in preparation.

[20] This has also been shown rigorously to be the case for the $1 d$ East model in D. Aldous and P. Diaconis, J. Stat. Phys. 107, 945 (2002) and the Kob-Andersen lattice model in C. Toninelli, G. Biroli and D.S. Fisher, cond-mat/0306746.

[21] Y. Brumer and D.R. Reichman, cond-mat/0306580.

[22] R. Yamamoto and A. Onuki, Phys. Rev. E 58, 3515 (1998); N. Lacevic, F.W. Starr, T.B. Schrøder, and S.C. Glotzer, J. Chem. Phys. 119, 7372 (2003).

[23] L. Berthier, Phys. Rev. E (Rapid Comm.) (in press, 2004), cond-mat/0310210.

[24] W. Kob and H.C. Andersen, Phys. Rev. Lett. 73, 1376 (1994).

[25] L. Berthier, Phys. Rev. Lett. 91, 055701 (2003). 